\title{
Mycobacteriosis Risk Factors
}

\section{Amirova T.H. ${ }^{1,}$ Petrov I.V. ${ }^{1,2}$ Petrova F.S. ${ }^{3,1}$ Petrova L.V. ${ }^{4,1}$ Sergeeva N.V. ${ }^{1}$}

\author{
Kholmatov A.V. ${ }^{1}$
}

\author{
${ }^{1}$ Mari State University, Yoshkar-Ola, Russia \\ ${ }^{2}$ Kazan State Medical University of the Ministry of Health of Russia, Kazan, Russia \\ ${ }^{3}$ Office of Rospotrebnadzor in the Republic of Tatarstan (Tatarstan), Kazan, Russia \\ ${ }^{4}$ Republican TB Dispensary, Yoshkar-Ola, Russia \\ *Corresponding author. Email: tanzilya.amirova.85@mail.ru
}

\begin{abstract}
Non-tuberculous mycobacteria are mainly saprophytes, but about 50 species are pathogenic to humans and cause various clinical manifestations. Currently, scientists have developed effective methods for identifying and diagnosing mycobacteriosis but have not fully confirmed risk factors for transmission to humans. This review focuses on summarizing various messages on this topic to identify possible risk factors for the transmission of atypical mycobacteria.
\end{abstract}

\section{Keywords: environmental objects, water sources, soil air, non-tuberculous mycobacteria}

\section{INTRODUCTION}

Currently, in the world, researchers have identified more than 200 species of Mycobacterium or non-tuberculous mycobacteria (NTMB) widespread in the environment. They are saprophytes or opportunistic bacteria belonging to the group of gram-positive, acid-resistant, non-sporeforming bacteria. However, 50 species of mycobacteria are causative agents of the disease of mycobacteriosis clinically and radiologically similar to tuberculosis [1-3]

NTMBs are represented by Enviromentalmycobacteria or atypical mycobacteria, this term was introduced by M. Pinner, 1935. The most characteristic difference between the NTMB group and the Mycobacterium tuberculosis complex is that NTMBs can hardly be transmitted from person to person [4-6].

The literature says about identifying NTMBs in drinking water, bottled water and also in biofilms of water pipes. Baths of beauty salons can have such forms as $\mathrm{M}$. avium complex (MAC), M. fortuitum, M. gordonae, $M$. simiae, M. mucogenicum, M. mageritense, M. smegmatis group, M. neoaurumlike, M. lentiflavum and fast-growing unidentified mycobacteria. Scientists have identified M. chelonae, M. chimaera, M. fortuitum, M. conceptionense, M. phocaicum in cooling towers and reverse water supply systems for heat, nuclear power plants, air conditioners, refrigeration units and cooling systems for electric generators [7].

\section{METHODS AND MATERIALS}

In the framework of this study, we analyzed the sources of medical information about risk factors for the transmission of mycobacteriosis by search electronic systems, including
PubMed (https://www.ncbi.nlm.nih.gov), Web of Science (http://webofknowledge.com), Scopus (https://www.scopus.com), eLIBRARY (https://elibrary.ru), ACP Journal Club (http://www.acpjc.org) and the British Medical Journal (https: // www. bmj.com).

We searched for information by keywords: nontuberculous mycobacteria, mycobacteriosis. Of the 327 sources found, the authors selected 170 sources based on keywords, after an analysis of the selected literature, the present study included 25 sources following the topic of the work.

\section{RESULTS}

Back at the beginning of the 21st century, Russian researchers took two hundred samples from environmental objects such as soil, manure, water, feed, etc. Examining 129 sputum isolates of patients with tuberculosis and 222 samples of tuberculin-sensitive animals, they found that atypical mycobacteria are widespread in the environment. Both tuberculin-sensitive animals and environmental objects, burdened with tuberculosis have the causative agents of cattle tuberculosis. Although the studied culture morphological and biochemical properties of 129 sputum isolates of patients with tuberculosis showed that 91 $(75.8 \%)$ and $29(24.2 \%)$ samples are M. tuberculosis and M. bovis, respectively, but 9 samples failed to be identified for various reasons [8].

Japanese researchers analyzed the molecular epidemiology of M. gordonae strains in patients and in a hospital setting. As a result, a total of 46 clinical strains were found in patients and 3 strains in the hospital environment of the medical centre. Using the genetic data of the 16S rRNA gene and hsp65PRA and pulse-field gel electrophoresis (PGE), they evaluated their intraspecific 
variability and epidemiology. Strains from six patients and environmental cultures showed different genes for sequencing 16S rRNA and hsp65PRA genes. However, PFGE analysis did not reveal a pseudo-outbreak and showed the presence of a polyclonal infection in one patient. In general, these results suggested the need to maintain effective environmental monitoring in the hospital and to conduct molecular epidemiological studies for infection control of M. gordonae continuously [9].

Many authors from Germany also indicated the transmission through medical equipment or in the provision of medical services.

In Germany, home care for hemato-oncological patients has now become preferable. In a prospective study, we studied tap water in the amount of 65 samples from the houses of these patients to assess the risk of infection with aquatic pathogens, such as non-tuberculous mycobacteria (NTMB), Legionella spp. and P. aeruginosa. The neutropenia of the patients or a suspected neutropenia period of at least 10 days was an additional special condition for selection. Required re-hospitalization patients were monitored for infection with the above pathogens over the next 3 months.

NTMBs were cultured in 62 samples $(95.4 \%)$ at home conditions in concentrations from 1 to $1000 \mathrm{CFU}$ in 500 ml. Most often, researchers identified facultatively pathogenic species $\mathrm{M}$. chelonae $(58.5 \%)$ and $\mathrm{M}$. mucogenicum $(38.5 \%)$. As a result, only one patient had M. chelonae. Although the risk of infection with mycobacteria in domestic conditions seemed low, the authors proposed preventive measures that are considered individually in patients with long-term immunosuppression, as well as in patients with long-term central vessel catheterization [10].

Other German authors reviewed the studies and as confirmation showed that invasive infections caused by $\mathrm{M}$. chimaera, part of the Mycobacterium avium-intracellulare complex, have been increasingly recorded in the last few years. Most infections occurred in patients undergoing cardiothoracic open chest surgery. Epidemiological and molecular studies have shown that the transmission of $\mathrm{M}$. chimaera occurs through intraoperative aerosols obtained from contaminated coolant heaters, i.e. devices used to turn on the extracardiac circuit in cardiothoracic surgery. Also, the researchers found mycobacteria data in vials for blood culture and in samples obtained surgically from diseased tissues during special microbiological tests to detect mycobacteria. The authors also suggested that the successful treatment of infections $M$. chimaera requires the removal of infected devices and long-term combination therapy with antimycobacterial drugs [11].

African researchers, together with scientists from Germany, conducted a study of Buruli ulcer, an advanced mycobacterial skin infection caused by $\mathrm{M}$. ulcerans. The authors studied the risk factors for this ulcer in Zio and Yoto areas of the coastal areas of the African country of Togo. It is necessary to add that the disease mainly affects the poor rural population, especially in areas with low standards of hygiene and sanitary services. Conducting a case-control study in both Zio and Yoto districts from
November 2014 to May 2015, the authors collected sociodemographic, environmental or behavioural data and conducted a logistic regression analysis to identify and compare risk factors between cases of the disease and control groups. As a result, they recorded 83 cases of the disease and proved that bathing in water from an open reservoir, insect bites near the river were potential risks for the occurrence of Buruli ulcer in the Zio and Yoto areas of the marine region in the south of Togo [12].

Authors from Greece have suggested that drinking water is a potential factor in the transmission of NTMB to humans. They studied hospitalized patients with NTMB infections together with samples of drinking water from 30 areas of residence during 2003-2013 and established the following: that the most frequently distinguished species of mycobacteria were $\mathrm{M}$. fortuitum, $\mathrm{M}$. gordonae, $\mathrm{M}$. peregrinum, M. chelonae, M. avium, and M. intracellulare. It was noted that 8 patients had tuberculosis with one NTMB, 15 patients had two types of NTMB simultaneously, while 1 patient had three different NTMBs. Besides, from 2010 to 2013, in 30 settlements, researchers selected, analyzed 3360 samples of drinking water and found $11.2 \%$ of mycobacteria. However, the correlation between mycobacteria found in patients and from samples of drinking water varied from mild to moderate, and thus the results showed that drinking water could be the cause of NTMB in humans [13].

Researchers from Italy studied the pseudo-outbreak of M. gordonae and obtained the following results. This mycobacterium was found in 7 samples from 497 of broncho-alveolar lavage after a bronchoscopy procedure in patients admitted to the hospital from January to April 2013. During this outbreak, specialists conducted clinical, epidemiological, environmental and molecular studies. As a result, none of the patients was diagnosed with mycobacteriosis of the lungs. Microbiological studies of water revealed M. gordonae in 3 samples: in tap water and the water supply channel of the washing disinfector. All strains were genotyped using pulse-field gel electrophoresis, which showed that only the patient strains had the same strip structure but there was no correlation with the strains isolated from water. Observation of the outbreak and strict adherence to the water treatment procedure and its supplies did not subsequently lead to the discovery of $\mathrm{M}$. gordonae in clinical respiratory samples. The authors made a recommendation: an outbreak of mycobacteriosis requires regular screening of tap water and endoscopic equipment to compare strains with each other [14].

A study in the Czech Republic showed that saprophytic mycobacteria could endanger health, especially for people with weakened immune systems. They included workers in the heavy industry and coal mines in this group, as their immunity is often weakened by exposure to dust, the fact of smoking and frequent respiratory diseases. This study dealt with the presence of NTMB in non-potable water used for the hygiene of workers at six large industrial enterprises and coal mines. For ten years, scientists studied 1,096 surface water samples. The most frequently identified species were M. 
gordonae, M. avium-intracellulare, and M. kansasii. The authors gave recommendations on precautionary measures to reduce the incidence of mycobacteriosis in workers at risk [15].

Scientists from France analyzed the cases of NTMB in the provision of cosmetic services and the quality of tap water used in these aesthetic centres. From 2001 to 2010, they registered 57 cases of mycobacteriosis, including 43 cases associated with services of aesthetic profile. The French public health authorities and the National Institute for Public Health Supervision recorded 40 cases of NTMB during non-surgical procedures performed by general practitioners in private conditions: mesotherapy, carboxytherapy and sclerosis of microvaricoses; 3 cases concerned surgical procedures-lifting and prosthetics of the mammary gland. As a result, an assessment of the quality of the provision of cosmetic services showed the lack of standard hygiene precautions and the improper use of tap water to clean injection equipment or disinfect the skin. Microbiological studies have confirmed the similarity of strains taken from patients and the environment in 16 cases after mesotherapy. M. chelonae isolated from tap water was similar to strains isolated in 11 cases from 16 cases [16].

In addition to the above, we would like to mention the possible concomitant factors of transmission of NTB.

Some foreign researchers studied the biofilm formed in tap water and noted that it was more often found in Mycobacterium avium complex, as well as fast-growing NTMB, such as $M$. abscessus, $M$. chelonae, $M$. mucogenicum, M. fortuitum. Biofilm in water supply systems (and other sources) is an essential component of natural cultivation of NTMB [17-22]. Biofilm is a thin layer of solid (pipe) and liquid (water) surfaces. The impermeable walls of mycobacteria contain fatty acids and wax and cause a hydrophobic surface that promotes bonding with solid substrates (e.g. pipes) in an aqueous medium, which leads to the persistence of mycobacteria and their resistance to rinsing with a water jet. Such biofilm features in almost all water collection systems and pipelines and probably provides nutritional support to microorganisms $[19,20]$. Since NTMBs are resistant to many disinfectants, their persistence can result from selection due to appropriate water treatment [20, 23, 24]. In addition, disinfectants located in tap water do not kill NTMB [19, 20]. Thus, Mycobacterium avium complex is many times more resistant to water chlorination than other microorganisms, this procedure even contributes to their selection. Such water contains hydrocarbons and biocides, which are substrates for the growth of mycobacteria. According to other data, mycobacteria of the environment mainly survive during the chlorination of tap water [25]. The widespread use of chlorination promotes the selection of more resistant strains and possibly explains the change of strains from M. scrofulaceum to M. avium, which, for example, are the main cause of cervical lymphadenitis in children [22].

\section{CONCLUSION}

This review presented a brief analysis summarizing the epidemiology and hygienic features of non-tuberculous mycobacteria that cause clinical manifestations in humans.

Thus, the world scientific environment has studies where the authors made an attempt to associate pathogenic NTMB strains with environmental objects, such as drinking water $[10,13,14]$, water of open reservoirs $[12,15]$, medical equipment $[9,10]$. However, it is not so simple and led us to the conclusion that other concomitant unaccounted factors increase the pathogenicity and/or invasiveness of non-tuberculous mycobacteria. Identification of these factors may be a solution for the development of effective preventive and antiepidemiological measures.

\section{REFERENCES}

[1] I.V. Petrov, M.O. Novikova, A.A. Almukhametov et al., Comparative Characteristic of ceses of mycrobacteriosis and tuberculosis among HIVinfected patients, Helix 8(1) (2018) 2988-2991.

[2] M. Cofini, P. Favoriti, F. Quadrozzi, Acute pancreatitis in pediatric age: our experience on 52 cases, Minerva Pediatr. 66(4) (Aug. 2014) 275-280.

[3] J.S. Laudelino, F.T. Farias Filho, A.F. Pedrosa Costa, V.M. Santos, Mycobacterium abscessus urinary tract infection: case report, Brazilian J. of Nephrol. (May 2019). DOI: 10.1590/2175-8239-JBN-2018-0260.

[4] T.F. Otten, Features of bacteriological diagnosis and etiotropic therapy of pulmonary mycobacteriosis, St. Petersburg, 1994.

[5] R. Prevos, T.K. Marras, Epidemiologi of human pulmonary infection with Nontuberculous Mycobacteria, In: A.H. Gwen, C.L. Daley, ed. Nontuberculous Mycobacteria: An Issue of Clinics in Chest Medicine, Elsevier Health Sciences, 2015, pp. 13-31.

[6] C.L. Daley, D.E. Griffith, Pulmonary non"tuberculous mycobacterial infections, Int. J. of Tubercul. and Lung Disease 14(6) (2010) 665-671.

[7] J.O. Falkinham III, Environmental Sources of Nontuberculous Mycobacteria, In: A.H. Gwen, C.L. Daley, ed. Nontuberculous Mycobacteria: An Issue of Clinics in Chest Medicine, Elsevier Health Sciences, 2015, pp. 35-41.

[8] I.V. Efendieva, R.A. Nuratinov, P.G. Umarova, Specific features of mycobacteria isolated from patients and animals with tuberculosis and fromenvironmental objects, Probl. Tuberkul. 5 (2001) 45-47.

[9] S. Yoshida, K. Suzuki, T. Iwamoto et al., Detection of molecular epidemiology of Mycobacterium gordonae isolates, Kekkaku 85(7) (Jul. 2010) 609-614.

[10] H. von Baum, M. Bommer, A. Forke et al., Is domestic tap water a risk for infections in 
neutropenic patients?, Infection 38(3) (Jun. 2010) 181-186.

[11] S.L. Becker, U. Schlotthauer, H.J. Schäfers et al., Epidemiology, clinical presentation, diagnosis and treatment of infections caused by mycobacterium chimaera, Pneumologie 73(8) (Aug. 2019) 474-481.

[12] I. Maman, T. Tchacondo, A.B. Kere et al., Risk factors for mycobacterium ulcerans infection (Buruli Ulcer) in Togo - a case-control study in Zio and Yoto districts of the maritime region, BMC Infect. Diseases 18(1) (Jan. 2018) 8-14.

[13] E. Dovriki, I. Gerogianni, E. Petinaki et al., Isolation and identification of nontuberculous mycobacteria from hospitalized patients and drinking water samples-examination of their correlation by chemometrics, Environ. Monit. Assess 188(4) (Apr. 2016) 247.

[14] L. Scorzolini, F. Mengoni, C.M. Mastroianni et al., Pseudo-outbreak of Mycobacterium gordonae in a teaching hospital: importance of strictly following decontamination procedures and emerging issues concerning sterilization, The New Microbiol. 39(1) (Jan. 2016) 25-34.

[15] V. Ulmann, A. Kracalikova, R. Dziedzinska, Mycobacteria in water used for personal hygiene in heavy industry and collieries: a potential risk for employees, Int. J. of Environ. Res. and Public Health 12(3) 2870-2877.

[16] C. Couderc, A. Carbonne, J.M. Thiolet et al., Nontuberculous mycobacterial infections related to esthetic care in France, 2001-2010", Med. Mal. Infect. 41(7) (Jul. 2011) 379-383.

[17] A. Al-Sulami, A. Al-Taee, Q. Wida'a, Isolation and identification of Mycobacterium avium complex and other nontuberculosis mycobacteria from drinkingwater in Basra governorate, Iraq, Eastern Mediterranean Health J. 18(3) (2012) 274-278.

[18] C. Armbruster, T. Forster, R. Donlan et al., A biofilm model developed to investigate survival and disinfection of Mycobacterium mucogenicum in potable water, Biofouling 28(10) (2012) 1129-1139.

[19] J. Falkinham, Ecology of nontuberculous mycobacteria - where do human infections come from? Seminars in Respirat. and Critical Care Med. 34(1) (2013) 95-102.

[20] J. Falkinham, Nontuberculous mycobacteria in the environment, J. Clin. Chest Med. 23 (2002) 529-551.

[21] S. Hernandez-Divers, D. Shearer, Pulmonary mycobacteriosis caused by Mycobacterium haemophilum and M. marinum in a royal python, The J. of the Amer. Veter. Med. Associat. 220(11) (2002) 1661-1663.

[22] H. Whiley, A. Keegan, S. Giglio, R. Bentham, Mycobacterium avium complex the role of potable water in disease transmission, J. of Appl. Microbiol. 113(2) (2012) 223-232.

[23] R. Revetta, V. Gomez-Alvarez, T. Gerke et al., Establishment and early succession of bacterial communities in monochloramine-treated drinking water biofilms, FEMS Microbiol. Ecol. 86(3) (2013) 404-414.

[24] H. Sebakova, F. Kozisek, Mudra R.J. Kaustova et al., Incidence of nontuberculous mycobacteria in four hot water systems using various types of disinfection, Canad. J. of Microbiol. 54(11) (2008) 891-898.

[25] T. Primm, C. Lucero, J. Falkinham, Health impact of environmental mycobacteria, Clin. Microbiol. Rev. 17 (2004) 98-106. 